\title{
Note sur le calendrier kasena
}

Note on the Kasena Calendar

Danouta Liberski

\section{(2) OpenEdition}

Journals

Édition électronique

URL : http://journals.openedition.org/span/590

DOl : 10.4000/span.590

ISSN : 2268-1558

\section{Éditeur}

École pratique des hautes études. Sciences humaines

\section{Édition imprimée}

Date de publication : 1 septembre 1986

Pagination : 101-120

ISSN : 0294-7080

\section{Référence électronique}

Danouta Liberski, « Note sur le calendrier kasena », Systèmes de pensée en Afrique noire [En ligne],

7 | 1986, mis en ligne le 05 juin 2013, consulté le 01 mai 2019. URL : http://journals.openedition.org/ span/590 ; DOl : 10.4000/span.590

(c) École pratique des hautes études 


\section{NOTE SUR LE CALENDRIER KASENA *}

\section{par}

\section{Danouta Liberski}

Les unités de temps du jour, du mois lunaire et de 1 'année solaire se structurent pour les Kasena sur le modèle du jour et de la nuit. Le mois lunaire (cana, "lune") est divisé en deux périodes, de 15 jours chacune, qui correspondent respectivement à la phase ascendante de 1 'astre lunaire (can punga, "1une blanche") et à sa phase décroissante (can yi kunu, "lune est sombre"). Chacune de ces périodes est également appelée cana et vaut "une lune"; toutes les enquêtes sur le calendrier ont débutées par l'affirmation "1a lune est de 15 jours". I1s compteraient done deux lunes pour une seule de nos lunaisons. Cependant, lorsqu'ils décomptent les mois lunaires qui composent une année, seule la phase ascendante de la Lune (la "lune blanche") est comptabilisée. Nous appelerons le premier compte.un compte "dédoublé" et le second un compte "par moitié". Pour nous expliquer cette opération logique qui pose que deux peut parfois être égal à un (que deux lunes de 15 jours valent un dans le décompte des mois), certains informateurs se sont référés à 1 'étrange couple du jour et de la nuit dans lequel le jour est compté comme un et la nuit, tout en étant distinguée du jour, ne s'y ajoute pas pour faire deux. Ils conclurent en disant qu'il en allait de même pour une lunaison et, nous y viendrons, pour une année. Préciser que la nuit ne forme pas une unité supplémentaire à celle du jour est eux, comme pour nous, une sorte de démonstration par 1'absurde : ils ne pour eux, comme pour. nous, une sorte de démonstration par 1'absurde: quand ils ont à évaluer le nombre de jours qui les séparent d'un événement (le marché par exemple), il ne leurvient pas à l'esprit de compter séparément les jours des nuits. Le jour ( $d \varepsilon, \mathrm{p} 1 . d a)$, lorsqu'il est une unité de Systèmes de pensée, Cahier 7, 1984 
compte est pour eux comme pour nous l'intervalle de temps qui sépare deux levers de soleil. Ainsi, de fait, tant le jour astronomique que la lunaison sont implicitement reconnus comme étant les unités de la mesure du temps (on aura très certainement vu que ne comptabiliser que la "lune blanche" pour une lunaison -- ou que la journée pour le jour -- revient arithmétiquement au même que prendre pour unité 1 'intervalle de temps qui sépare deux nouvelles lunes -- ou deux levers de soleil. Le calendrier kasena, malgré les particularités du comptage, reste en accord avec le temps astronomique). Par l'évocation du couple du jour et de la nuit, les informateurs semblaient dès lors vouloir souligner que toute unité de temps (que ce soit celie du jour, du mois ou encore de l'année) est un espace de temps qui n'est pas uniforme. Ainsi du jour astronomique qui en tant qu'unité de compte vaut un et qui est formé d'une journée et d'une nuit. La manière un peu particuIière dont les Kasena comptent apparaît alors comme une façon remarquablement économique de dire que toute unité de temps est faite d'une dualité (le compte "par moitié", en distinguant ce qui se compte de ce qui ne se compte pas, maintient un dire sur 1 'existence de deux temps contrastés à l'intérieur de 1 'intervalle de temps envisagé tout en énonçant l'identité numérique de cet intervalle). Le temps de l'action rituelle est, chez les Kasena, essentiellement déterminé en fonction de ces temps alternés qui, nous allons le voir, ne sont pas mêmement qualifiés.

Indépendamment de la distinction entre un temps de la "lune blanche" et un temps de la "lune sombre", il existe d'autres découpes temporelles du mois lunaire qui sont basées principalement sur la position qu'occupe la Lune dans le ciel au moment où elle devient visible. Les noms de certaines de ces divisions du mois lunaire font référence à 1 'aspect de la Lune -- comme "blanche", "sombre", "dévoilée" -- mais d'autres -- comme "faible" et "forte" -- qualifient la durée de visibilité de l'astre lunaire pendant la période ainsi dénommée et non l'intensité de sa luminosité. Ajoutons encore que les indications sur le nombre de jours pour chaque période et sur la position de la Lune dans le ciel sont. celles données par les informateurs lors d'un discours diurne (la position de l'astre ayant été indiquée par un geste du bras) et ne proviennent pas de constatations empiriques, mêrne si celles-ci ont cherché à vérifier celles-là. 
Ils distinguent ainsi : 1) can dunga nungi, "nouvelle lune sortie", le premier jour où la Lune se laisse apercevoir, un bref moment, à 1 'ouest et le jour suivant; 2) can bwona, "1une faible", les 3ième, 4 ième et 5 ième jours pendant lesquels $1 \mathrm{a}$ Lune, lorsqu'elle apparaît, est toujours dans le premier quart de la moitié ouest du ciel; 3) can dan, "lune forte", période de 5 jours décomptés à partir du jour où 1'astre devient visible alors qu'il est dans le second quart de la moitié ouest du ciel; 4) can punga, "Iune blanche", période de 5 jours encore, décomptés à partir du jour où la Lune se laisse voir au méridien

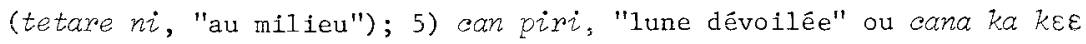
kabiro kwaga, la "lune est partie derrière le mur" (i.e. à l'est) ou encore cana sugi kalwe, la "lune qui effraye les singes", le quinzième jour alors que la lune surgit à l'est, pleine et rouge d'être en opposition avec le soleil; 6) cana z'u kuri, la "lune est rentrée au-dessous", période de 5 jours lors desquels la Lune apparaît de plus en plus tard après le coucher du soleil; lorsqu'elle se lèvera -- à l'est -- on dira can puli, la "Iune pousse"; 7) can yii kunu, la "1une est devenue sombre", période de 10 jours lors desquels on n'aperçoit plus la Lune de toute la nuit. Les premiers jours de cette dernière période, on peut encore voir, dans cette heure qui sépare 1'aube de 1'aurore, un mince croissant se profilant à l'est. Les Kasena diront alors que la "lune a rejoint le soleil" (cona ka de wia) et qu'elle suivra pendant le jour le chemin du soleil. (Le souci de périodiser par des laps de temps de 5 jours ou d'un multiple de 5 nous surprend, les Kasena ne possèdant pas d'unité de temps comparable à la semaine de cinq jours. I.'unité supérieure au jour et inférieure au mois lunaire est un cycle de trois jours qui correspond à 1'intervalle de temps qui sépare deux marchés tenus dans un même village. Ces trois jours sont décomptés à partir du jour où se tient le marché. Ce qui laisse entre chaque marché deux jours que 1 'on distinguera en se référant aux marchés qui se tiennent ces deux jours là dans les villages voisins (1)).

Il existe un certain décalage entre ces découpes temporelles qui sont opérées par un discours quotidien et celles qui sont pertinentes pour la détermination du temps d'effectuation d'un rite. Ces dernières se réduisent au nombre de quatre : 1) can dunga nungi, 5 jours; 2) can punga, 10 jours; 3) cana z'u kumi 5 jours; 4). can yi kunu, 10 jours. 
Ces quatre divisions du mois lunaire vont rythmer l'activité rituelle selon un code pré-établi et connu de tous. Il est remarquable de constater -- mais peu aisé d'interpréter -- que la plupart des indications que nous possèdons sur 1 a détermination temporelle des rites dans une lunaison se présentent sous la forme d'interdictions, comme si le code ne prenait en considération que les temps où les rites ne pouvaient avoir lieu et non ceux où ils doivent obligatoirement prendre place. La seule indication positive, en effet, serait celle concernant un rite lié à la première grossesse d'une femme (ba ling kane, "ceindre la femme") qui doit toujours avoir lieu dans la période de la "nouvelle lune sortie" (on nous a parfois précisé qu'il ne pouvait toutefois s'agir que des 3ième, 4ième et 5ième jour de la nouvelle lune). Ce rite entre dans la catégorie des rites de passage. Accompli au troisième mois de la grossesse, il vise dit-on à empêcher que la jeune femme avorte. Mais c'est aussi lors de ce rite que son état est officiellement reconnu et, plus exactement dit, qu'il est pour la première fois dévoilé (2). Un interdit très marqué pèse sur l'annonce pub1ique d'une grossesse, qu'elle soit ou non la première; la femme attendra en général le troisième mois pour déclarer qu'elle est enceinte et cette annonce, en aucun cas, ne pourra se faire dans le temps de la "lune sombre" ( une fausse couche en résulterait). Comme pour la première grossesse, il faudra attendre le temps de la "nouvelle lune sortie".

Cette injonction faite à la femme enceinte ne prend son sens que si elle est mise en relation avec ce qui ne peut advenir dans le temps de la "lune sombre". Les indications sont là plus nombreuses, la dernière période de la lunaison apparâ̂ssant comme un temps durant lequel toute une série de rites et d'activités rituelles ne peuvent se dérouler sans voir leur efficacité s'annuler. c'est ainsi que ne peuvent s'actualiser les dix derniers jours de la lune les rites pour la sortie, après une réclusion d'un mois, d'un chef nouve1lement élu; les rites pour la construction d'une nouvelle maison ("sortir" est l'expression qu'utilisent les Kasena pour désigner un déménagement, que celui-ci soit collectif ou individue1); les rites des secondes funérailles et ceux de levée du deuil qui marquent la fin de la période d'errance pour un mort et sa séparation d'avec le monde des vivants. Enfin, dans le domaine des activités agricoles mais néanmoins 
rituelles, s'il est permis de battre le mil, on ne pourra cependant pas séparer le grain du son. La nature de ces rites et de ces activités rituelles nous fera dire que ce qui ne peut se produire lorsque la. "lune est devenue sombre", c'est tout événement qui s'apparente à une naissance (et à ce qui s'ensuit, c'est-à-dire la séparation de 1 'enfant d'avec son placenta). Il est pour nous évocateur que la naissance d'un enfant est jugée peu propice pour l'enfant si elle advient en cette période. Décrit comme un petit être qui sera toujours faible et souffreteux, il offre l'image d'un prématuré (3). Nous nous demandons alors si les Kasena, en s'interdisant de produire certains événements au temps de la "lune sombre", événements qui par ailleurs ont toutes les caractéristiques d'une naissance rituelle, ne cherchent pas à éviter cela qui arrive à l'enfant né dans cette période, à savoir $1 \mathrm{a}$ prématuration, l'interruption avant terme d'une gestation.

Revenons au premier rite que nous avons évoqué, le rite de "ceindre la femme". Que sa détermination temporelle relève d'une même logique, cela peut à première vue surprendre : ne s'agissait-il pas là d'un rite lié à une grossesse et non à une naissance? Il faut se souvenir cependant que ce rite, par les gestes effectués et les paroles prononcées, insiste sur le dévoilement : il rend "visible" ce qui était jusqu'alors "caché" (tu). On peut penser alors que pour les Kasena, 1 'annonce d'une grossesse n'appartient plus au temps de la gestation mais relève déjà d'un temps de la naissance. Que la rupture de l'interdit de temps soit formulée en termes de "fausse couche" renforce 1 'hypothèse que ce qui est en jeu dans ce rite, c'est l'évocation d'une naissance : 1'annonce faite en un temps non adéquat provoque l'apparition dans la réalité de l'événement évoqué.

Nous retrouverions donc chez les Kasena une logique des temps cosmiques qui serait très proche -- quoique formulée à l'aide de représentations différentes -- de celle dont M.Cartry a démontré qu'elle était à l'oeuvre dans le calendrier gourmantché (4). Cet auteur a montré, entre autres choses, comment pour les Gourmantché les périodes de la nouvelle lune et celle de la lune noire correspondaient respectivement à un temps de la "naissance" et à un temps de la "gestation". Le premier de ces temps semble moins bien marqué chez les Kasena mais 
cela, indépendamment des lacunes de notre enquête, est peut-être dû au fait que les Kasena expriment par des prohibitions ce que les Gourmantché formulent à 1 'aide d'injonctions positives : interdire que des événements de l'ordre d'une naissance adviennent au temps de la "lune sombre" revient sans doute au même que de prescrire ces mêmes événements au temps de la nouvelle lune mais la négation étant plus difficile à interpréter que l'affirmation, un doute subsiste.

Ajoutons enfin qu'il existe des activités rituelles totalement indépendantes des divisions du mois lunaire et même dirons-nous du temps astronomique. La divination semble pouvoir se pratiquer tous les jours du mois, tous les mois de l'année, à toutes heures du jour et, en théorie du moins, à toutes celles de la nuit. Seule la mort -- proche ou effective -- d'un membre du lignage du devin empêche ce dernier de répondre à la demande d'une consultation. La mort, dit-on, "ferme les yeux du vogo" (i.e. le principe divinatoire).

Les divisions de l'année

Lorsque 1 'unité de temps envisagée est celle de l'année, nous retrouvons un mode de comptage très semblable à celui rencontré pour une Iunaison. Une fois encore l'accent est mis sur l'alternance entre deux temps contrastés et une attention moindre, à première vue, est prêtée à 1 'année comme unité de mesure du temps.

L'année (bini, plur. bino) est divisée en deux périodes, appelées également bini, de 7 lunaisons chacune : les 7 lunes de yade (période correspondant approximativement à la saison des pluies et dont nous n'avons pu jusqu'à présent trouver 1 'étymologie du nom) et les 7 lunes de tepunga, "terre blanche" (période correspondant à la saison sèche (5)). La proposition : "1'année kasena est de 7 lunes" fut entendue au moins aussi souvent que celle affirmant que "la lune est de 15 jours". Certains Kasena, soucieux d'insister sur le contraste entre ces deux "années" vont jusqu'à dire que seules les 7 lunes de yade sont dotées d'un nom et que les 7 lunes de tepunga, bien que décomptées, ne sont pas nommées. Les sept noms donnés sont tous fonction des travaux agricoles et de la nature des récoltes en ces mois, exception faite d'une seule, faa cana, dont la traduction littérale "lune des récoltes" ne 
renvoie pas à l'activité effective de cette période (1es récoltes ont lieu le mois suivant) mais dénote le rite par lequel s'achève cette lunaison : le rite de $b a d i$ fa $\alpha$, "ils mangent les récoltes", appelé

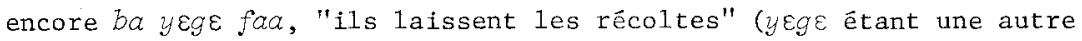
façon de dire "sacrifier", kane) qui est tout à la fois un marqueur temporel important pour l'année et le signal attendu pour pouvoir débuter les récoltes proprement dites.

Les noms des sept lunes de yade, en respectant l'ordre dans leque1 elles nous ont été déclinées, sont les suivantes :

1. duni cana,

2. pare cana,

3. care cana,

4. gware cana,

5. Kamana cana,

6. faa cana, "lune des semailles" qui prend approximativeplace entre la mi-mai et la mi-juin.

"lune du premier sarclage" (15 juin-15 juil.)

"lune du mil hâtif" (15 juili.-15 aôut)

"1une du second sarclage" (15 aôut-15 sept.)

"1une du maîs", appelée également nonguri lizeo cana, "lune des arachides hâtives". Période pendant laquelle commencent les récoltes secondaires : maiss, arachides hâtives, patates douces, haricots (15 sept.-15 oct.)

"lune des récoltes". Bien qu'elle précède les récoltes des principales céréales (mil, sorgho), elle est considérée comme un mois d'abondance (les récoltes secondaires ont eu lieu). C'est pourquoi on l'appelle encore : won da an bu cana, "lune Qui n'est pas mon enfant?", la réponse sous-entendue étant : "personne". I1 y a tant à manger que tout un chacun aura sa part de nourriture dans les maisons où il s'arrêtera, comme s'il était un enfant de 1 a maison ( 15 oct. -15 nov.)

7. mena zegem cana, "lune du mil coupé". Mois où débuteront donc les récoltes de mil et de sorgho et qui ne s'achèveront que vers $1 \mathrm{a}$ fin du mois suivant, soit vers le début de janvier puisque cette lune-ci prend approximativement place entre la mi-novembre et la mi-décembre.

Avec cette dernière lune se termine la période yade dans le même temps où, pour les Kasena, les travaux agricoles prennent officiellement fin : après, dit-on, chacun est libre et il faudra compter 7 lunes pour semer (duni) à nouveau.

Les Kasena compteraient donc "deux" (années) quand nous, nous compterions "un" (une année civile). Mais à cette proposition que nous leur soumettions, nos informateurs se mirent à sourire : à nouveau, 
ils invoquèrent le couple du jour et de la nuit. Et de leur dire, il ressortait que seules les 7 lunes de yade formaient 1 'unité du compte, les 7 lunes de tepunga s'y ajoutant sans former une unité supplémentaire. Nous retrouverions donc, pour 1 'année, ce que nous avions appelé "un compte dédoublé" (1'année est de 7 lunes et il y a deux "années" dans une de nos années civiles) et "un compte par moitié" (seule l'année yade est comptabilisée). Il est certes plus difficile, lorsque l'unité de temps est 1 'année, de s'assurer dans 1 'après-coup du terrain que le "compte par moitié" a quelque usage pour les Kasena : à la différence des mois lunaires, nous $n$ 'avons pas trouvê trace d'une comptabilité portant sur plusieurs années successives.

Mais les propos ici rapportés, tenus par deux devins au cours d'enquêtes distinctes, n'empêchent pas que par ailleurs 1'on puisse obtenir des noms pour. Ies mois lunaires de la période tepunga. La contradiction n'en est pas une si $1^{\prime}$ 'on admet qu'une fois encore la division de l'année en deux "années" d'un nombre égal de lunes chacune, loin de prétendre être une mesure du temps, vise à exprimer une opposition signifiante entre deux temps qui s'alternent. De fait, les listes que nous avons recueilities comportent -- les erreurs et les oub1is mis à part -- 10 noms de mois lunaires auxquels, nous a-t-on toujours précisé, il faut ajouter "trois" lunes que l'on ne nomme pas (6). En regard de 1 'affirmation précédente, c'est une lune de manque mais du point de vue de notre calendrier grégorien, c'est une lune de trop (du moins si elle est comptabilisée chaque année). On aura compris que les chiffres "7" et "3" n'ont pas ici valeur numérique (7). Mais on ne peut négliger le fait que le chiffre "3" permet peut-être aux Kasena de tenir en un seul compte le calendrier des années ne comportant que 12 lunes et celui des années où, pour réajuster l'année lunaire à l'année solaire, il faut ajouter une lune supplémentaire (une lune tous les trois ans environ si 1 'on considère que $1 \mathrm{e}$ calendrier de base comporte 12 mois lunaires). Cette hypothèse se verrait peut-être confirmée par la place qu'occupent. ces lunes non nommées dans la succession des mois. D'après nos 1 istes, les "trois lunes sans nom" (en fait deux selon notre manière d'envisager les divisions de 1 'année) sont décomptées à partir de la lune qui succède à la lune de fac. Arrêtons-nous ici un instant. Nous venions d'apprendre que la lune qui suit la lune de faa appartenait à la période yade, el1e en était même la dernière, et qu'elle était nommée ("1a 
lune du mil coupé"). Mais ceux-1à même qui soutenaient que cette lune était sans nom ne remettaient pas en cause que tepunga recommencait après les récoltes effectives, soit après cette première lune qu'ils ne nommaient pas (et donc entre la mi-décembre et la mi-janvier). Les lunes non nommées sont dès lors respectivement la dernière lune de yade et la première de tepunga : elles sont en quelque sorte les lunes qui font la charnière entre les deux périodes. C'est peut-être là une autre façon de rendre bien distincts les deux temps qui constituent l'année solaire tout en se ménageant la possibilité d'ajouter une lune les années où, comme disent les Kasena, "yade est en retard".

Après ces lunes intermédiaires se succèdent :

warro cana, "lune du froid" (15 janv.-15 févr.).

gun'kogo viu cana, "Iune du vent du kapokier séché" (15 févr.-15 mars).

Iwem wia cana, "lune de la grosse cigalè", litt. cigale du soleil, (15 mars-15 avril).

Leri cana, "lune du net toyage (des champs)" (15 avril-15 mai).

Malgré le fait que la notion de nouvelle année ne soit pas absente du vocabulaire kasem (ben'dore) ni absente l'idée d'un recommencement (après les récoltes), nous hésitons à y voir un début d'année -- au sens où nous entendons cette unité de temps -- dans la mesure où le terme qui désigne $I$ 'année (bini) serait, nous $I^{\prime}$ avons vu, mieux rendu par "période de 7 lunes" que par "année". Nous n'avons par ailleurs aucune trace de l'existence d'un rituel lié au changement d'année, du moins dans I'état actuel de nos enquêtes.

Par contre, il est deux "fêtes" rituelles qui, chaque année solaire, viennent re-fixer le calendrier lunaire des Kasena. Ces deux fêtes sont liées au cycle de la graine -- elles précèdent respectivement les semailles et les récoltes -- et c'est par elles que commence et presçue s'achève la séquence temporelle yade, découpée avec tant d'insistance par les deux devins (elle est celle que $1^{\prime}$ on compte et les mois qui la composent sont nommés). Ces fêtes constituent bien deux marqueurs du temps en ce sens que les deux mois lunaires où elles devront prendre place sont, à notre connaissance, les deux seuls mois du calendrier dont la détermination dans le temps fait l'objet d'un décret ritualisé. Il revient au "mâ̂tre de la terre" (tega'tu) de décider et d'annoncer, après consultations divinatoires, que le temps des semailles (duni cana) 
ou le temps de $f a \alpha$ ( $f \alpha \alpha$ cana) est arrivé. Pour le premier de ces temps, c'est encore le tega'tu qui domera le signal pour que dans chaque maison s'effectue auprès des autels d'ancêtres le sacrifice des semences prélevées sur la récolte précédente (8). La détermination de la "lune des semailles" s'opère sur la base de critères écologiques, astronomiques et météorologiques. Les deux premiers types de critères sont reconnus de tous, comme le bourgeonnement de certains arbres (saborsina, Acacia dudgeoni; sampunu, ind.) et le coucher héliaque des pléiades, ci'biE'ru, "la mère des poussins" -- lorsque ces dernières disparaissent à l'ouest au moment oì le soleil se couche, la période yade a commencé et la nouvelle lune qui suivra sera appelée duni cana. L'observation du régime des pluies, par contre, est uniquement du ressort du "maître de la terre" : à lui revient de décréter que la pluie qui vient de tomber annonce la "pluie des semailles" (duni dua). Il serait trop long de dire ici pourquoi et en quoi c'est le tega'tu qui détient ce rôle déterminant. Mais retenons que se retrouvent chez les Kasena des représentations liées au cycle du mil qui sont très proches de celles qui furent attestées chez les Dogon. Le mil possède une âme, disent les.Kasena : mem'joro, mil/âme (joro étant le même terme qui est utilisé pour désigner 1 'âme des hommes). Conservée dans "la maison de $w \varepsilon^{\prime \prime}$, le dieu solaire, durant l'intervalle de temps qui sépare deux périodes de semailles, "I âme du mil" doit être "attrapée" et redescendue sur terre pour le temps de son séjour terrestre, lequel s'achève lors durite de $b a$ di faa qui la renvoie à son lieu d'origine. Le sacrifice des semences (kane dwi) qu'effectue le "mâtre de la terre", au moment des semailles, auprès de la Terre et des tangwana (lieux sacrés) cherche, dit-on, à faire descendre la pluie qui entraînera avec elle "l'âme du mil". Voici les termes en lesquels cela nous fut raconté par le tega'tu de Kaya (9) :

"(...) il faut donner (i.e.sacrifier) aux semences là pour qu'elles attrapent le souffle, qu'elles attrapent le corps frais (la santé). Quand je sacrifie, j'appelle comme ça (...). Quand le moment arrive, ce sont les tangwana qui montent dans la maison de $w \varepsilon$ pour prendre le mil. Ils montent et décident avec $w \varepsilon ;$ w enlève (une quantité déterminée) et leur donne, ils attrapent et descendent avec (le mil). Qu'est-ce qui fait que la pluie ne vient pas à tepunga et qu'elle vient à yade? C'est comme ça. C'est les tangwana qui montent et tous, avec $w \varepsilon$, ils décident et maintenant, ils attrapent la pluie et ils descendent pour que les êtres humains mangent les choses là (...). Si ca pousse hors de la terre 
et si la pluie ne vient pas, ils vont tous mourir. Si la pluie vient, ça va pousser, comme ça, frapper partout, partout. C'est la pluie qui "commande" (10) le mil et son âme. Comme le mil a quitté la maison de $\omega \varepsilon$, est venu là, et la pluie a fini de pleuvoir, ça reste maintenant; nous avons attrapé et nous mangeons maintenant. Comme ils (les aînés de lignage) sont allés sacrifier tous leurs sacrifices (pour faa), qu'ils ont fini, l'âme du mil retourne dans la maison de wह".

Le deuxième temps dont la détermination fait 1'objet d'un "décret", celui de faa, est une période lors de laquelle est imposé un silence rituel. En ce temps délicat où le mil, encore sur pied, "accouche", on ne peut frapper les tambours, piler le mil à la tombée de la nuit, danser à l'enterrement d'une personne âgée, ni même crier ou siffler. Toute infraction à l'interdit ferait tomber le mil et ouvrirait le pays aux épidémies (les maladies les plus fréquemment invoquées étant 1a "rougeole" -- kəcuə -- et 1a "variole" -- tambol b (11)). Le fauteur de trouble, quant à lui, serait "maudit" par la terre et les tangwana. C'est encore un temps où, plus que jamais, l'interdit de verser le sang sur la terre est en viigueur. Les blessures que s'occasionnent les cultivateurs en travaillant aux champs exigent 1'immédiate intervention du tega'tu afin qu'il effectue le sacrifice pour "ramasser le sang" (ba $p \varepsilon$ jana). Veiller à ce que ces interdits soient respectés par tous est du ressort du "maître de la terre" mais cette fois associé en cette tâche au chef (peo), rare sinon unique occasion où I'un et I'autre conjugent leurs charges (12). Le mois s'achève par le rite de $b a$ di faa qu'il revient au chef de commencer. Il versera successivement sur ses autels d'ancêtres et sur le kwara (fétiche lié à la chefferie) une libation d'eau mélangée à de la farine du nouveau mil. A sa suite, le tega'tu fera une libation sur ses propres autels d'ancêtres avant: de se rendre "là où la terre respire" (l'autel de.la terre pour l'ensemble de la communauté) pour une nouvelle libation. Ce dernier rite nous fut décrit par le tega'tu de Kaya en ces termes:

\footnotetext{
"Je vais, je laisse sur la terre. Come la terre et tous les tangwana sont une seule chose, je donne à la terre, je dis qu'elle prenne avec ses tangwana; eux aussi qu'ils attrapent et montent dans la maison de we, pour qu'ils demandent encore cemême mil là pour cultiver encore; pour qu'ils demandent le corps frais, qu'ils donnent aux êtres humains ("les personnes noires") pour qu'elles mangent foo avec saveur".
} 
Le double signal -- donné successivement par le chef et le tega'tu -- enclenchera des sacrifices de même nature qui seront effectués tout le long du réseau lignager : chaque aîné de lignage maximal, chaque aîné de segments de lignage et ainsi du reste en suivant l'ordre de la segmentation jusqu'à chacun des maîtres de maison "laisseront fad" (i.e. sacrifieront $f a \alpha$ ) sur leurs autels d'ancêtres respectifs. L'âme du mil étant ainsi retournée en son lieu d'origine, les récoltes proprement dites peuvent commencer. Lorsque ces dernières seront terminées, un dernier rite prendra place. Vers la mi-janvier, les cultivateurs iront individuellement, au rythme de 1 'achèvement de leurs travaux des champs, "saluer" et "remercier" celui des "maîtres de la terre" ou des tangwam'tina (plur. de tangwam'tu, charge liée à un tangwam) dont relèvent rituellement leurs terres de culture (13). Ils emmèneront avec eux des paniers emplis du mil de la nouvelle récolte, des houes, de la cola, du tabac que le tega'tu ou le tangwam'tu sacrifiera discrètement à 1 a terre ou au tanguam au fur et à mesure des visites. Tout comme le rite de $b a d i$ faa, cette salutation rituelle a pour but, dit-on, de renvoyer l'âme du mil dans la "maison de $\omega \varepsilon^{\prime \prime}$; mais alors que le premier est un marqueur temporel très net qui engage 1 'ensemble de la communauté sous I'égide du chef et du tega'tu, le second s'échelonne dans la seconde moitié de janvier, n'exige aucun "signal" de commencement et s'opère individuellement (les membres d'une même maisonnée pouvant avoir des champs qui relèvent de I'autorité rituelle de différènts tega'tina (plur. de tega'tu) ou tangwam'tina. Il est néanmoins possible qu'il s'agisse des deux parties d'un seul et même rite (mais il faudrait, pour pouvoir 1'affirmer, poursuivre I'enquête). Quoi qu'il en soit, constatons que la période "charnière" des lunes non nommées s'insère très exactement entre ces deux rites associés aux récoltes. Après le rite de salutation des "mâttres de la terre", on dira que tepunga a vraiment commencé; des travaux et des occupations d'une autre nature vont pouvoir débuter.

Le regroupement des mois lunaires en deux grandes périodes suit, certes, le rythme d'une nature "en travail" pendant la "saison des pluies" et au repos à la "saison sèche". Ce rythme, pour les Kasena, s'inscrit dans le ciel : la voie Lactée (sisem, "limite") divise la voûte céleste en un ciel de tepunga et un ciel de yadદ (pour une observation faite en janvier, alors que la Voie Lactée court approximative- 
ment du nord au sud, les deux "ciels" étaient respectivement la partie orientale du ciel, "la plus blanche" ajoute-t-on, et sa partie occidentale). Observant les déplacements de cette trace étoilée au cours de 1'année solaire, les Kasena veulent y voir la preuve d'une lutte permanente entre les deux ciels. Le ciel de tepunga, disent-ils, "repousse" celui de yade à l'ouest : lorsque la Voie Lactée est visible à 1'ouest peu de temps après le coucher du soleil (vers le mois de mai), tepunga a envahi toute la voûte céleste, son temps s'achève et celui de yadદ va commencer. Le mouvement s'inverse alors, le ciel de yade "repoussant" peu à peu tepunga vers 1 'est : lorsque la Voie Lactée sera visible à l'est, toujours peu après le coucher du soleil (vers la fin décembre et le début de janvier), yade est entièrement déployé dans les cieux, son temps est achevé, la saison sèche a commencée.

L'alternance des saisons imprime sa cadence, on le sait, aux travaux quotidiens. Mais elle impose également son rythme aux activités rituelles. Nous venons de voir que ces dernières étaient entièrement centrées sur le cycle de la graine lors de la période yadદ. La période tepunga, elle, se caractérise par une intense activité rituelle, individuelle et collective, dont il serait difficile de résumer la nature d'un seul trait. Mais il existe des rites qui ne peuvent se dérouler qu'au temps de tepunga et qui, dans une société aussi peu portée aux festivités collectives que celle des Kasena, frappent par le nombre de personnes qu'ils engagent dans leur procès : nous parlons des rites pour les secondes funérailles et des rites pour la levée du deuil.

Cette bi-partition temporelle de l'activité rituelle en un cycle de la graine et un cycle des morts ne répond pas à la seule nécessité pratique mais renvoie, croyons nous, à une logique des temps cosmiques que les Kasena nous invitent à déchiffrer en nous référant à 1'alternance du jour et de la nuit.

Les divisions du jour

Une imagerie mythique attribue le lever du jour et la tombée de la nuit respectivement à 1 'ouverture et à la fermeture du fruit de calebassier cosmique que $\omega \varepsilon$, le dieu solaire, a divisé, en un temps anté-humain, en une moitié céleste et une moitié terrestre. Que le jour 
succède à la nuit, cela répéterait 1 e geste mythique qui permit 1 'avènement des hommes dans le monde. La langue porte la trace de cette imagerie : "à demain" se dit en kasem ka poore, abréviation de tega ka na poore, "la terre s'est ouverte" (i.e. quand la terre se sera fermée puis ouverte une nouvelle fois). En outre, si les différents moments de la journée sont tous désignés par des termes qui font référence au soleil, ceux de la nuit sont exprimés par des syntagmes formés sur 1e mot "terre" : la nuit se dit tete, qui est le redoublement du radical te sur lequel est construit tega, la "terre"; le milieu de la nuit est appelé à Koumbili te seru, "terre resserrée" et enfin, les premières lueurs de 1 'aube sont désignées tega ka kaara, "la terre se déchire". Les petits matins blancs et brumeux du mois de janvier quand le soleil se voile de la poussière de sable qu'amènent les vents du nord, on dit encore : tega kuni, "la terre est sombre". Ainsi, si l'on en croit 1 a langue, un règne de la terre commence dès le moment où l'astre solaire n'est plus visible. Il est dès lors tentant de penser que 1 'alternance du jour et de la nuit correspond pour les Kasena à 1 'alternance des règnes des deux puissances autour desquelles s'organise leur cosmogonie : $\omega \varepsilon$, le nom de leur dieu solaire est la transformation phonétique de wia, le soleil (la transformation phonétique est une marque de respect qu'utilisent les cadets lorsqu'ils prononcent le nom de leurs ânés) et tega est tout à la fois le nom de la puissance et le terme qui désigne la terre dans son aspect matériel.

Un début de confirmation à cette hypothèse pourrait être donné par les faits suivants. Lorsqu'au cours d'une consultation divinatoire, le consultant cherche à déterminer le temps d'effectuation du rite qui vient de lui être prescrit, il opérera toujours son choix en ne retenant des différents moments possibles de la journée que celui du lever du soleil ( $w \varepsilon$ puli "1e soleil pousse") et celui de son coucher (we zoore, "le soleil est rentré"). D'après les consultations que nous avons recueillies, il ressort que les rites 1 iés à des demandes individuelles faites aux ancêtres sont toujours temporellement déterminés au temps de la nuit commençante. Il est par ailleurs des rites pour lesquels il n'est pas besoin de consultations pour savoir qu'ils doivent prendre place au lever du soleil. Tel est le cas du rite effectué pour l'entrée d'un enfant de quelques mois dans son 1 ignage agnatique. Ce rite, de type initiatique, met en jeu un fétiche lié à la procréation du lignage 
(bu'nyua,"le bouilion de l'enfant"). La très belle prière qu'adresse au fétiche 1 'officiant du rite fait entendre que dans le même temps où 1 'on cherche à placer 1 'enfant sous la protection du fétiche ("il entre dans le bu'nyua") , on demande à ce dernier de faire advenir de nouvelles naissances dans le lignage. Les termes de la prière rappellent les paroles prononcées par le tega'tu lors des rites pour les semailles et les récoltes. Donnons en ici un extrait, en précisant qu'il s'agit d'une prière qui fût énoncée pour l'entrée de deux enfants dans le lignage maximal du chef:

"Tu es le mâtre de maison. Nous, nous voulons te donner ces personnes. C'est toi qui dis que tu es le vieux marigot, que tu n'es jamais à sec et toi maintenant tu es asséché, tu es complètement arrêté. Nous, nous voulons te donner ces personnes là. Celui-ci (le premier des enfants), aujourd'hui, est à présent devenu la personne de notre dwi ("semence", "clan") (...). Vois l'eau que je te donne. Parce que tu es le vieux marigot qui n'est jamais à sec, toi, tu vas monter dans la maison de we, tu demandes le corps frais, tu donnes au mur là (le mur de la maison); je ne veux pas que l'eau manque à l'intérieur de toi (...). Prends et atteins les ancêtres de cette maison, atteins leur kwara (fétiche lié à la chefferie), atteins leur dieu (wc), atteins leur pum (tas d'ordures devant la maison du chef), atteins leur tangwam, atteins toute l'étendue de la terre de Kaya. Tu cherches le corps frais, tu cherches les êtres humains qui ont le souffle dur (la vie longue) et tu mets sous ce mur (...)".

Nous ne pouvons malheureusement pas, dans le cadre de cet article; 1ivrer la totalité de cette prière ni donner le commentaire qu'elle exige. Mais retenons ici que pour les Kasena, le mil, les êtres humains comme "toutes les choses du monde" sont d'une seule et nême semence ( $(w i)$, celle de la pulpe du fruit de calebassier cosmique (zum'zone) que we retient dans la moitié céleste du fruit et que les hommes par les sacrifices et les prières cherchent à faire descendre dans la moitié terrestre pour qu'elle se transforme "en mil, en êtres humains, en toutes les choses du monde".

Les éléments que nous venons d'évoquer sur 1a répartition temporelle des rites au cours d'une journée semblent indiquer dès lors que le lever du soleil comme marque du début du jour -- et du règne du dieu solaire -- est le temps durant lequel doit prendre place les rites qui visent à "faire descendre" un peu de la semence céleste alors que le coucher du soleil, comme marque du début de la nuit -- et du règne de la terre -- est un temps réservé aux ancêtres. (Dans le petit 
village de Koumbili, le coucher du soleil déclenche immanquablement la scène suivante : le groupe des vieux qui depuis le matin se tenaient avec le chef autour de "l'arbre de la chefferie" se lèvent brusquement et sans mot dire, d'un seul et même mouvement, quittent le dessous de 1 'arbre. Ils laissent la place, disent-ils, aux ancêtres du chef qui, chaque soir, à la tombée de la nuit, reviennent s'asseoir aux lieux qu'ils occupaient de Ieur vivant).

Au début de notre propos, nous disions que 1a manière selon laquelle les Kasena enregistraient le temps dénotait un discours qui posait que tout intervalle de temps pris comme unité de mesure était formé d'une dualité. Au terme de cette première investigation sur le calendrier, nous pouvons ajouter que par leur double facon de compter, les Kasena nous invitent à distingurer entre, d'une part, la nécessité d'être dans un temps quantifié (nécessité à laquelle répond le compte "par moitié") et, de 1'autre, la nécessité de déterminer le temps d'effectuation d'un rite et donc, la nécessité d'être dans un temps qualifié (à laquelle répond le compte "dédoublé). L'un et l'autre de ces temps sont fonction du temps astronomique. Mais tandis que pour le premier, les Kasena se réfèrent au retour des astres à la même place (deux levers de soleil, deux nouvelles lunes), pour le second de ces temps, ils ne retiennent que les places opposées qu'occupent les astres dans le ciel au cours d'un intervalle de temps donné (1e lever et le coucher du soleil, la nouvelle lune et la lune dans son dernier quartier, le lever des Pléīades à l'est et leur coucher à l'ouest, les déplacements de la Voie Lactée). Le temps quantifié est basé sur l'immuabilité, le temps qualifié est structuré sur 1 'alternance.

Le jour et la nuit, la lune blanche et la lune sombre, yade et tepunga sont utilisés par les Kasena comme les éléments d'un code : nous avons cherché à pointer quelques-unes des significations supportées par ces éléments. Ce.n'est là encore qu'une esquisse, la connaissance des temps d'effectuation pour d'autres rites devant nous aider à mieux préciser 1 a nature des temps cosmiques qui à chaque fois s'a1ternent dans les trois unités de temps envisagées. Mais il serait sans 
doute vain de vouloir réduire à une seule équation les natures de ces temps cosmiques : l'alternance, parce qu'elle est une structure, aggrège en ses pôles des signifiés divers qui prennent leur valeur par opposition à d'autres signifiés et non dans une correspondance terme à terme.

Danouta Liberski

L.A. 221

\section{NOTES}

(*) Au nombre de 95.000 environ, les Kasena occupent, dans 1a partie méridionale du Burkina Faso (ex Haute-Volta), la région intérieure de $1 \mathrm{a}$ boucle de $1 \mathrm{a}$ Volta Rouge et s'étendent, au-delà de la frontière qui sépare le Burkina du Nord du Ghana, jusqu'au $11^{\circ} 30$ de latitude Nord. Linguistiquement, ils se rattachent à la sous-famille gurunsi des langues voltaīques. Population d'agriculteurs, ils tirent 1 'essentiel de leur subsistance de plusieurs variétés de mil et de sorgho. Leurs société est patrilinéaire et le noyau de l'unité résidentielle est constitué par 1 a descendance agnatique du fondateur. Une logique segmentaire très proche de celle décrite par M.Fortes pour les Tallensi sous-tend le système lignager. Ils connaissent une double hiérarchie du pouvoir : celle des chefs sacrés (peo) et celle des "maîtres de la terre" (tega'tu). Le territoire d'une communauté relève en général d'un seul tega'tu et d'un peo (il existe cependant des villages sans chefferie propre) mais il est toujours morcelé en plusieurs terres relevant chacune de l'autorité rituelle d'un tangwam'tu (charge liée à un tangwam,lieu sacré souvent entendu comme "autel de la terre" dans la littérature ethnographique). Le matériel utilisé ici a été recuei11i, sauf mention contraire, à Pô et à Kaya, communautés de la région orientale du pays kasena, au cours de trois missions (nov.81-févr.82; nov.82-mai 83; janv.-févr.85) dont les deux dernières furent respectivement financées par le L.A.221 et le Laboratoired'Anthropologie sociale; le L.A.221. Pour des raisons de facilité de lecture et de facilité de frappe, nous avons adopté une transcription linguistique simplifiée : la nasale vélaire est notée ng et la nasale palatale ny; nous $\mathrm{n}$ 'avons pas distingué les voyelles de 2 ième degré d'aperture ( $l$ et $v$ ) des voyelles de troisième degré d'aperture (e et $o$ ) ni la voyelle d'aperture moyenne $(\partial)$ de celle d'aperture maxima $(a)$. 
(1) A la différence de ce que G. Le Moal a constaté pour les Bobo du Burkina, il ne semble pas qu'il y ait, pour l'ensemble du pays kasena, trois marchés de référence; les constellations sont toujours locales : ainsi pour le village de Kaya, les deux jours qui séparent deux marchés de Kaya sont respectivement appelés "1e jour de marché de Songo" et "le jour de marché de Tangassogo" (deux villages immédiatement voisins de celui de Kaya). Mais nous ne connaissons pas, pour le moment, les composants de chacune des constellations du pays kasena. Notons encore que tout un chacun connait par ailleurs les jours des marchés des trois villages les plus importants du pays (Pô, Tiébélé, Paga). Entendons qu'ils connaissent les "vrais" jours de ces marchés, ces derniers étant devenus permanents. Nous ne pouvons dire s'ils servaient d'étalon pour le cyle des trois jours.

(2) Ce rite se déroule comme suit : une fille mariée du lignage du mari (1a kadiko), choisie par divination, se rend de nuit -- personne ne doit la "voir" -- dans la maison où repose la jeune femme. A 1 'aube, la kadiko prélève un peu de cendres dans l'un des foyers, se dirige vers 1 a case de la jeune femme et 1'appelle. Lorsque celle-ci, feignant la surprise, passe le seuil, la kadiko souffle en sa direction la cendre qu'elle tenait sur la paume ouverte de la main, et dit simplement : a piri mon, "je t'ai surprise" (piri a le triple sens de "surprendre", "rendre visible ce qui était caché" (dévoiler) et "blanchir". Le rite, par les signifiants qu'il utilise, joue sur les trois significations du terme : "la jeune femme surprise", "blanchie par la cendre" et "dont l'état jusqu'alors tu est révélé"). La kadiko attachera ensuite aux reins de la jeune femme un fil de coton noir qui sera dénoué après la délivrance et passé au cou du nouveau-né.

(3) Différent est le statut et le destin d'un enfant né le premier jour de 1 a nouvelle Lune. De ce dernier on dit "qu'il est venu avec la lune". I1 aura toujours pour nom acana ou kacana (les complétants la/ et / ha/ précisent le sexe de l'enfant : respectivement garçon ou fille). Sa "faiblesse" est attribuée à son lien beaucoup trop étroit avec la Lune. Les gestes rituels qui sont accomplis à la naissance d'un tel enfant semblent venir dire qu'il faut remettre une certaine distance entre l'astre lunaire et lui afin d'éviter qu'il ne voit ses forces décroître en proportion inverse à l'accroissement de la Lune chaque mois. Le premier de ces gestes rituels sera d'accrocher au cou de l'enfant, dans les premiers jours qui suivent la naissance, un pendentif fait d'un morceau de cuivre jaune auquel le forgeron aura donné pour l'occasion la forme d'un mince croissant de Lune. Ce geste s'éclaire d'un autre : une opération rituelle homologue est réalisée pour un enfant dont on s'aperçoit qu'il possède tous les traits physiques de celui de ses parents qui appartient au même sexe que lui. Le fer d'une pointe de flèche appartenant au père ( $s^{\prime i l}$ s'agit d'un garçon), un morceau de l'une des calebasses neuves de la mère ( $s^{\prime} i 1$ s'agit d'une fille) sont dérobés à l'insu de leur propriétaire et transformés par le forgeron en petits bracelets qui seront passés au poignet du nouveau-né. Le commentaire donné alors suggérait que trop de ressemblance avec qui vous avait engendré entrâ̂nait le dépérissement et empêchait la croissance. 
(4) Exposé du 20 juin 1981 dans le cadre du L.A.n²21, résumé dans le Rapport Scientifique du L.A.n $221,1981-1983$, p.7.

(5) Ces notations mises entre parenthèses n'ont de valeur qu'indicative, les périodes yade et tepunga ne coincidant qu'approximativement avec ce que les géographes appellent la saison des pluies et 1a saison sèche. Doté d'un climat soudanien, le pays kasena connait une saison des pluies de 4 à 5 mois (de la mi-mai à la mi-octobre) et une saison sèche de 6 mois environ (de la mi-novembre à la mi-avril). Entre ces deux saisons s'intercalent des régimes transitoires qui présentent des alternances de saison sèche et d'incursion d'air humide (cf. G. Pallier, Géographie générale de Za Haute-Volta, publication de I'U.E.R. des lettres et Sciences Humaines de l'Université de Limoges avec le concours du C.N.R.S., 1978, 241 p.). La différence entre les saisons climatiques et les divisions kasena de $l^{\prime}$ année est relativement importante puisque selon ces dernières, yade s'étend de la mi-mai à la mi-décembre (7 mois) et tepunga de la mi-décembre à la mi-mai (5 mois). On verra que pour les Kasena la détermination de ces périodes n'est pas fonction du climat mais de la nature des travaux qui s'y déroulent.

(6) Ces informations concernent la région orientale du pays kasena (Pô et Kaya). Lors d'un premier séjour en une région différente

(Koumbili,à 1 'ouest du pays), nous avions recueilli les noms pour 12 mois lunaires (sans mention d'une 13ième lune). Les difficultées rencontrées à Pô et à Kaya pour obtenir l'énumération des mois témoignent, certes, d'une certaine désagrégation de la connaissance quant à ce propos mais 1 'existence de lunes comptées mais non nommées est bien, croyons-nous, une caractéristique inhérente au calendrier de l'est. Notons encore que malgré les nombreuses divergences culturelles et les différences dialectales entre ces deux régions, les recoupements entre la liste de Koumbili et celles de la région orientale font apparaître 6 noms de lunes identiques dont la plus importante : faa cana qui sert de marqueur temporel pour le début des récoltes.

(7) Ces chiffres dont on connait la récurrence et la valeur symbolique en Afrique de l'Ouest sont chez les Kasena les "nombres d'or" pour toute séquence rituelle, ainsi de même pour le chiffre 4.

(8) Nos renseignements sur ce rite sont peu nombreux, le tega'tu auquel nous nous adressions se plaignant qu'aujourd'hui que la pratique de semer à sec avait été introduite, les aînés de lignages ne venaient plus le voir pour lui demander si la pluie à venir était bien "la pluie pour semer". Il semblerait que le signal donné par lui consistait d'une part en la réponse faite à la demande des aînés -- codée comme suit : pour une réponse négative "laisse cette pluie pour construire ta maison" et pour une réponse positive "va dire à tes enfants de cultiver pour toi" -- et d'autre part, il devait être le premier à "ouvrir la terre" (o poro te te'kan) ou "blesser la terre" (o eane te'kan) en pratiquant sur son champ les poquets dans lesquels sa femme allait enfouir les semences. Il est encore le premier à devoir "sacrifier la semence" (kane dwi) en versant une libation d'eau de farine de mil sur ses autels d'ancêtres d'abord, sur la terre ensuite. 
(9) Dans une publication en préparation, nous donnerons la version intégrale, accompagnée d'une traduction littérale, des récits et des prières dont nous avons livré ici les extraits.

(10) Le terme utilisé est te qui donne le radical / tu/ avec lequel se construisent les syntagmes qui désignent toute charge rituelle.

(11) Nous devons la détermination des plantes et des maladies au 1 inguiste E.Bonvini, in "Les noms de plantes :et les noms de maladies", inédit, s.1.s.d., C.N.R.S.T., Ouagadougou.

(12) Bien qu'il ne semble pas (ou plus) que les rites effectués avant la période des semailles et avant celle des récoltes prennent chez les Kasena 1'allure de ces festivals agraires décrits par M.Fortes pour les Tallensi, nous voulons voir un trait commun dans cette alliance du chef et du tega'tu au moment du rite de faa. Tout se passe comme s'il fallait suspendre toute activité sonore mais aussi toute dualité, toute séparation des tâches pour permettre à la terre d'accomplir son dur travail de parturition.

(13) Les champs situés en brousse peuvent relever de l'autorité rituelle de tega'tina ou de tangwam'tina appartenant à des communautés voisines. Ils auront en un temps antérieur effectués les rites nécessaires pour que le cultivateur puisse inaugurer son nouveau champ. 